\title{
Single Step Undirected Reconfigurable Networks
}

\author{
YOSI BEN-ASHER $^{\mathrm{a}, *}$ and ASSAF SCHUSTER ${ }^{\mathrm{b}}$ \\ ${ }^{a}$ Dept. of Comp. Sci., Haifa U, Haifa, Israel; \\ ${ }^{\mathrm{b}}$ Department of Computer Science, Technion, Haifa 32000, Israel
}

(Received 5 May 1997)

\begin{abstract}
The reconfigurable mesh (RN-MESH) can solve a large class of problems in constant time, including problems that require logarithmic time by other, even shared memory, models such as the PRAM with a similar number of processors [3]. In this work we show that for the RN-MESH these constants can always be reduced to one, still using a polynomial number of processors. Given a reconfigurable mesh that computes a set of values in constant time, we show that it can be simulated by a single step reconfigurable mesh with maximum size that is polynomial in the size of the original mesh. The proof is constructive, where the construction of the single step RN-MESH holds for the relatively weak undirected RN-MESH model. In this model broadcasts made on buses arrive at all nodes that belong to the undirected connected component of the transmitting processor. A result similar to the one that is obtained in this work was previously obtained for the directed reconfigurable mesh model (DRN) [5]. However, the construction for the DRN-MESH relies on the fact that the buses are directed, and thus cannot be applied to the undirected case. In addition, the construction presented here is simpler and uses significantly fewer processors than the one obtained for the DRN-MESH.
\end{abstract}

Keywords: Reconfigurable networks, reconfigurable mesh, constant time, undirected connectivity

\section{INTRODUCTION}

The basic idea of the reconfigurable mesh, or, the RN-MESH, is to enable flexible connection patterns in the network by allowing processors (nodes) to connect and disconnect their adjacent edges in various patterns (referred to as states). The various network connection patterns may be used by the algorithm to accelerate computations. Informally, this is due to sampling the connectivity in these patterns, which is induced by-and may indicate of $-\mathrm{a}$ lot of the (input) values stored by the network processors.

At the beginning of each broadcasting step (or simply, a step) during the execution of a program, each processor of the RN-MESH fixes its basic state. Figure 1 shows a set of 15 different states that are generated by a node of the RN-MESH by connecting the four internal nodes in different patterns. Broadcasts can be performed by disconnected internal nodes. Note that each state has a different number of broadcast possibilities. For

*Corresponding author. e-mail: yosi@mathcs.haifa.ac.il 
example, in Figure 1 internal nodes $l_{i, j}$ and $r_{i, j}$ may transmit in node $s 1$, whereas $u_{i, j}$ and $d_{i, j}$ may transmit in node $s 2$. Adjacent edges that were connected by processors are viewed as (hardware) connected, so that they form an integral bus. Any processor connected to an edge participating in the construction of a certain bus may choose to listen to any incoming or passing message transmitted on that bus, where some conflict resolution policy should be applied when multiple transmissions are made at the same time on the same bus.

A large volume of constant time and pseudoconstant time algorithms were devised for the reconfigurable mesh (RN-MESH) model, e.g. $[4,6$, 10]. Perhaps the most typical examples of these RN-MESH algorithms are the constant time sorting algorithms [4,9,12,14,17]. Obviously, there is a constant bounding the number of steps required for sorting $n$ numbers by an $n \times n \mathrm{RN}$ MESH. Since each of these steps involves a (possibly global) broadcast, it is important to determine how many of them are required, or in other words, how far can we push-down the running time of the algorithm. In this paper we make a first step towards resolving this question by showing that the constants involved in reconfigurable mesh algorithms can always be reduced at the expense of enlarging the mesh. Given an $n \times n \mathrm{RN}$ MESH that computes a set of values in a constant number of steps, we construct a single step RNMESH with size that is still polynomial in $n$ and that simulates the operation of the original mesh.
Another motivation for this work comes from the search for efficient design methodology for VLSI circuits. In this respect the ability of the RNMESH to compute in a single step may lead to the efficient construction of such circuits. One general idea in this direction is to develop fast RN-MESH algorithms and use an automatic construction (such as the one described in this paper) to transform them into single-step RN-MESHes. Single-step RN-MESH programs can be realized as asynchronous circuits that are a lot faster and easier to fabricate than their synchronized counterparts.

The results in this work applies to the undirected RN-MESH, and may be seen as the undirected counterpart of the result in [5] which shows that a constant time directed reconfigurable mesh (DRNMESH) can be simulated by a single step DRNMESH. The directed version of the reconfigurable mesh assumes that the underlying mesh is a directed graph where transmission follow the direction of the edges (see [3] for precise definitions). As it turned out, the directionality of the edges is essential for the construction in [5], hence it cannot be used for the undirected model. Note that it was shown in [3] that the directionality of the edges makes the DRN-MESH more powerful than the RN-MESH with respect to the set of problems that can be computed in constant time.

The result for the undirected model improves upon the construction of the DRN-MESH in the following aspects:
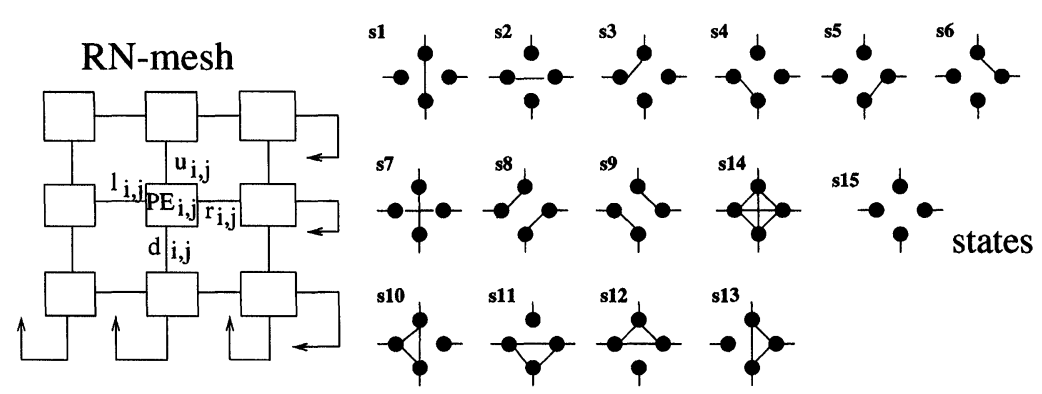

FIGURE 1 The RN-MESH and its basic set of states that may be assumed by each of the processors at every step. 
- The size increase is exponential, rather than doubly exponential in the directed case. More precisely, for an $n \times n$ mesh executing a $T$-steps program the size of the single step mesh increases by a factor of $n^{T}$ for the RN-MESH as compared to a factor of $n^{2^{T}}$ for the DRNMESH.

- The construction for the DRN-MESH is complex and involves reduction to non-deterministic branching programs, while the current construction for the RN-MESH requires no intermediate models.

- The size increase of the single-step RN-MESH is proportional to the number of events where the next state of a processor is a result of its failing to detect any broadcasts in the previous step. As this situation occurs rarely in RN-MESH algorithms, then the expected blowup in size of the single step RN-MESH is further reduced.

The proposed construction is based on a special representation of any given RN-MESH computation using two basic operations:

$\mathrm{UC}_{s, t}(G)$ undirected connectivity, so that $\mathrm{UC}_{s, t}$ $(G)=1$ iff there is a path from $s$ to $t$ in the undirected graph $G$.

$\overline{\mathrm{UC}}_{s, t}(G)$ the complement of $\mathrm{UC}_{s, t}(G)$, so that $\overline{\mathrm{UC}}_{s, t}(G)=1$ iff $s$ and $t$ reside in different connected components in the undirected graph $G$.

Connectivity queries, as many researches have noted, are natural (i.e., simple and fast) for RNMESHes. In fact, since the network is used for computation, the RN-MESH may be viewed as if it were a programmable connectivity oracle. Computing $\overline{\mathrm{UC}}_{s, t}(G)$ is more difficult; in fact, the fast computation of these functions is related to open problems in complexity theory (see [3]). For instance, in the case of the DRN-MESH [5] we had to use Immerman's algorithm [7] for directed unreachability. Similarly, the proposed construction in this work for the undirected RN-MESH uses the result of Nisan and Ta-Shma [13], namely, an "almost monotone" reduction of $\overline{\mathrm{UC}}_{s, t}(G)$ to $\mathrm{UC}_{s, t}(G)$. (A monotone circuit is a boolean circuit of polynomial size, that does not use negations. An almost monotone circuit is a monotone circuit that can use the complements of the input variables.)

The stages of the construction are as follows:

1. A formal model describing the operation of the RN-MESH is given in Section 2. This model is based on the notion of labeled graphs, where edges are selected according to boolean edgefunctions that are associated with some of the edges. It is shown that a given RN-MESH of size $n \times n$ which computes a set of values in $T$ steps (denoted by $\mathrm{RN}_{n}^{T}$ ), is equivalent to a sequence of $T$ labeled graphs $G^{1}, \ldots, G^{T}$, where the inputs to the edge-functions of $G^{t}$ are connectivity queries of the form $\mathrm{UC}_{s, u}\left(G^{t-1}\right)$ and $\overline{\mathrm{UC}}_{s, v}\left(G^{t-1}\right)$.

2. The main theorem, proved in Section 3, shows that labeled edges of $G^{t}$ can be replaced by copies of $G^{t-1}$ or by a labeled graph that computes $\overline{\mathrm{UC}}_{s, t}\left(G^{t-1}\right)$, except for $G^{1}$ whose edge-functions queries the input variables.

Repeating this process recursively yields that $G^{1}, \ldots, G^{T}$ can be simulated by a single labeled graph $\hat{G}$. In turn, $\hat{G}$ can be simulated by a single step RN-MESH, thus proving the main result.

3. The existence of a labeled graph that computes $\overline{\mathrm{UC}}_{s, t}\left(G^{t-1}\right)$ is discussed in Section 4 , and is based on the reduction presented in [13].

The formal RN-MESH model (i.e., the labeled graph) that we consider uses single-bit buses and processors. Only ' 1 ' signals can be transmitted, and a ' 0 ' signal is detected if no ' 1 ' signal was transmitted. For a certain input of size $n$ bits, one bit of the input is assumed to be given at each of a subset of $n$ processors. This, however, is not a mandatory restriction: the construction applies also to a model in which input bits may appear during different time steps of the algorithm at different locations.

This labeled graph representation is simple enough to allow the formal treatment of the model, yet powerful enough to efficiently simulate common variations of reconfigurable meshes, including for example the RN [4], PARBS [18], 
RMESH [11] and RMBM [15]. In particular, the ability to simulate the word model by the bit model has been addressed in $[1,8]$ and requires no further discussion. Other aspects of RN-MESHes, including: "empty" signals, collision detection, and local memory, can be easily simulated as described in [5].

\section{A GRAPH REPRESENTATION FOR RECONFIGURABLE MESH}

In this section we give a precise definition of the RN-MESH using the concept of labeled graphs. We show that a set of $T$ labeled graphs can be used to represent the computation of a $T$ steps $\mathrm{RN}$ MESH. The reason for giving this model is that using it we can reduce the reconfiguration operation to the problem of computing connectivity and un-connectivity in labeled graphs.

The first step towards a formal description of the RN-MESH is to replace the "mesh of switching processors" by a graph representation. This can be done if we use the complement-graph (where nodes and edges switch roles) of the mesh (see Fig. 2). In the complement mesh each processor (PE in Fig. 2) is represented by four nodes and six internal edges, and the reconfiguration or the change of states is accomplished by simply selecting some of the internal edges (see Fig. 2, right side). The $\bullet$ nodes are auxiliary nodes (one for each PE) used to simulate broadcasts of ' 1 ' values, as follows. Broadcasting is performed by selecting the internal edge that connects the $\bullet$ node of the transmitting PE with the internal node that represents an edge of the original mesh which leads to the direction in which broadcasting is performed. For example, in Figure 2, the PE in the upper-leftmost corner is transmitting to the left (out-link $d$ ), which is performed in the complement mesh by selecting the edge which connects the internal node $\bullet$ with the internal node $d$.

Broadcasts in the complement mesh are therefore a matter of connectivity. Namely, a processor receives a message $M$ iff there is a connected path of selected edges in the complement mesh leading from the $\bullet$ node of the processor which transmits $M$ to one of the nodes of the current processor. An example for the edge selection process is depicted in Figure 2. Note the relation of the edges selected in the complement mesh and the resulting connected components to the configuration in the original RN-MESH. For instance, note the path leading from the $\bullet$ node of the PE at the secondrow leftmost-column to internal nodes $r$ and $o$ of the bottom-row rightmost-column $\mathrm{PE}$, which represent a corresponding broadcast and receive operations in the original RN-MESH.

We remark that the number of edges needed to represent a processor will turn out to be a major factor in the calculation of the size of the single step RN-MESH used to simulate the $T$ steps of the given R-MESH. It is thus desirable to minimize this number, and this is the main motivation for using the complement mesh.

We now turn to the precise definition of the edge selection process. The proposed representation uses the graph representation of the RN-MESH,

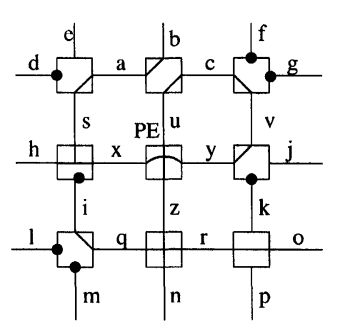

RN-MESH

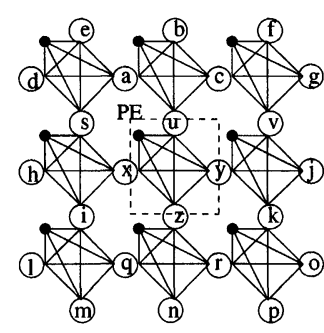

COMPLEMENT -MESH

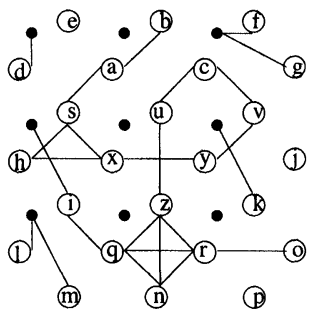

SELECTING EDGES

FIGURE2 Simulation of a reconfigurable mesh by a complement graph and edge-selection. 
as described above, with a labeling of its edges used for the edge selection process. The RNMESH executing a $T$ steps computation is represented by a sequence of $T$ labeled-graphs $G^{1}, \ldots, G^{T}$, where each edge in $G^{t}, t=1, \ldots, T$ may be labeled by an edge-function that is used to select this edge at the $t$-th step.

Definition 2.1 A labeled graph $G=\langle V, E\rangle$ is an undirected graph with a special node $S \in V$ and input variables $x_{1}, \ldots, x_{n}$. Some of the edges in $G$ may be labeled by edge-functions, where an edgefunction $\left(f_{e}\right)$ is an almost monotone boolean formula, i.e., a boolean function with a set of boolean inputs $x_{1}, \ldots, x_{n}$ and their negations $\bar{x}_{1}, \ldots, \bar{x}_{n}, e . g ., f_{e}=x_{5} \vee \bar{x}_{8}$. A boolean value is associated with every node $v \in V$ such that $v=1$ iff there is a path from the special node $S$ to $v$, and such that all the edge-functions along that path have been evaluated to one.

The importance of the labeled graph representation is that each step of the RN-MESH can be represented by a labeled graph $G^{t}$, such that the edge-functions of $G^{t}$ determine the possible state configurations that may take place in the $t$-th step of the RN-MESH. In order for this construction to work properly, the inputs to the edge-functions of $G^{t}$ must be the boolean values associated with $G^{t-1}$,s nodes, so that the reconfiguration at step $t$ will use the results obtained in the previous step.

Let $R N_{n}^{T}$ denote an $n \times n$ RN-MESH executing a $T$ steps computation. A labeled graph $G^{t}$ corresponding to one step of $\mathrm{RN}_{n}^{T}$ is the complement-graph of an $n \times n$ mesh as described earlier (see Fig. 2), where each processor is represented by three nodes (including the signal node $\bullet$ ) and ten edges, $3 n^{2}$ nodes and $10 n^{2}$ edges in all. In addition, each $G^{t}$ has one global signal node $S$ used to transmit ' 1 ' signals. This node is connected to all the $\bullet$ nodes of all PEs.

We now describe the set of nodes, edges, and edge-functions in the labeled graph representation that are associated with each processor $P E_{i, j}$ in the sequence of labeled graphs $G^{1}, \ldots, G^{T}$. For simplicity we sometimes omit the superscript ${ }^{t}$.
- Two internal nodes $l_{i, j}$ and $u_{i, j}$ (standing for 'left' and 'up'). The PE also has two other internal nodes, namely $u_{(i+1) \bmod n, j}$ and $l_{i,(j+1)}$ $\bmod n$, which for reasons of clarity we associate with neighboring PEs.

- Six internal edges used to represent states of the original processor $\mathrm{e} 1_{i, j}=\left\langle l_{i, j} u_{i, j}\right\rangle$, e $2_{i, j}=$

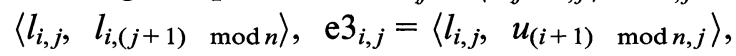
$\mathrm{e} 4_{i, j}=\left\langle l_{i,(j+1) \bmod n}, u_{i, j}\right\rangle, \mathrm{e} 5_{i, j}=\left\langle l_{i,(j+1) \bmod n}\right.$, $\left.u_{(i+1) \bmod n, j}\right\rangle$, and $6_{i, j}=\left\langle u_{i, j}, u_{(i+1) \bmod n, j}\right\rangle$.

- Four signal-edges used to send a ' 1 ' signal from the $\bullet_{i, j}$ node to any of the other internal nodes $\mathrm{e} 7_{i, j}=\left\langle\bullet_{i, j}, u_{i, j}\right\rangle, \mathrm{e} 8_{i, j}=\left\langle\bullet_{i, j}, l_{i, j}\right\rangle, \mathrm{e} 9_{i, j}=$ $\left\langle\bullet_{i, j, u(i+1)} \bmod n, j\right\rangle$, and $\mathrm{e} 10_{i, j}=\left\langle\bullet_{i, j}, l_{i,(j+1)}\right.$ $\bmod n\rangle$.

- An edge $e ?_{i, j}$ of a labeled graph $G^{t}$, for $t \in\{2, \ldots, T\}$, may be labeled by a boolean edge-function of the form

$$
f_{\mathrm{e} ?_{i, j}}^{t}=l_{i, j}^{t-1} \wedge \overline{u_{i, j}^{t-1}} \vee u_{i+1 \bmod n, j}^{t-1} \wedge \overline{l_{i, j+1 \bmod n}^{t-1}}
$$

where $u_{i, j}^{t-1}$ and $l_{i, j}^{t-1}$ are boolean variables (to be defined next) associated with the appropriate nodes of $G^{t-1}$.

- The edges of $G^{1}$ may be labeled by edge functions that are simply the input bits $x_{i}$ (or their negation) given to each processor of the RN-MESH prior to its computation, i.e., either $f_{\mathrm{e} ? i, j}^{1}=x_{i}$ or $f_{\mathrm{e} ? i, j}^{1}=\bar{x}_{j}$.

- A boolean variable associated with a node $u_{i, j}^{t}$ or $l_{i, j}^{t}$, is also denoted by the index of that node (it should be clear from the context when we refer to the node itself $u_{i, j}^{t}$ or to its associated boolean variable). The value of a boolean variable is true, i.e., $u_{i, j}^{t}=1$ or $l_{i, j}^{t}=1$, iff there is a path in $G^{t}$ from the global signal node $S$ to either of $u_{i, j}^{t}$ or $l_{i, j}^{t}$, and so that the value of all the edgefunctions along that path is ' 1 '.

- The values computed by $R N_{n}^{T}$ with inputs $x_{1}, \ldots, x_{n}$ after $T$ steps are the values taken by the boolean variables that are associated with the nodes of $G^{T}$.

We remark here that in the proof of our main theorem we will have queries asking whether certain 
edge-functions are evaluated to ' 1 '. As described above, each of these queries involves solving a connectivity problem from the $S$ node. If we did not have the global $S$ node, we could have answered the same queries by solving connectivity problems from all of the - nodes. However the latter option would imply a blowup in the size of the final simulation, as there are many $\bullet$ nodes. This is the reason to the somewhat-artificial addition of the $S$ node.

Consider, for example, processor $\mathrm{PE}_{0,0}$ of $G^{5}$ in Figure 3. The edge-functions are set such that the next state (at step $t=5$ ) will be either $\langle u p$ broadcast, down-broadcast, right-left $\rangle$ or $\langle$ left-up, right-down $\rangle$, depending on the values of $u_{0,0}^{4}$ and $l_{0,0}^{4}$. The un-labeled edges are not included in the configuration (or equivalently, are assumed to be labeled by the constant ' 0 '), regardless of the value of the input variables. Note that if $u_{0,0}^{4}=0$ and $l_{0,0}^{4}=0$ then the processor will broadcast on $u_{0,0}^{5}$ and $u_{1,0}^{5}$. These broadcasts will in turn determine the value of nodes that are connected to them by a path whose edge functions (at Step 5) have been evaluated to 1 .

Since the original RN-MESH program $\mathrm{RN}_{n}^{t}$ and its corresponding sequence of labeled graphs $G^{1}, \ldots, G^{T}$ are dual representations we refer in the sequel to both of them as the RN-MESH computation. In general, the computation of a given RN-MESH is a sequence of $T$ steps, in which we evaluate all edge-functions and determine the boolean variables $u_{i, j}^{t}$ and $l_{i, j}^{t}$ for the next step. These variables represent broadcasts received by processors, while edge-functions reflect reconfigurations. Eventually the values of the edgefunctions and the variables $u_{i, j}^{t}$ and $l_{i, j}^{t}$ depend on input values, hence the recursive chain of dependencies between edge functions and boolean variables is well-defined. Any computation of an RN-MESH can be represented in this way, and is implied by the simulation of each configuration of the RN-MESH as a proper choice of the edge functions in $G^{t}$. In particular, a simple induction on $t$ can be used to show that the values of $u_{i, j}^{t}$ and $l_{i, j}^{t}$ are identical to the broadcasts detected at $\mathrm{PE}_{i, j}$ at step $t$ of the RN-MESH.

Consider, for example, the well-known parity computation of two bits by a $2 \times 3 \mathrm{RN}-\mathrm{MESH}$ as described in Figure 4. Note that up-going signals on the upper row are redirected to the bottom row, so that the modulo-two counting operation is realized. The edges of every processor are marked for convenience and are represented on the right side of Figure 4. First, the input bits are broadcast

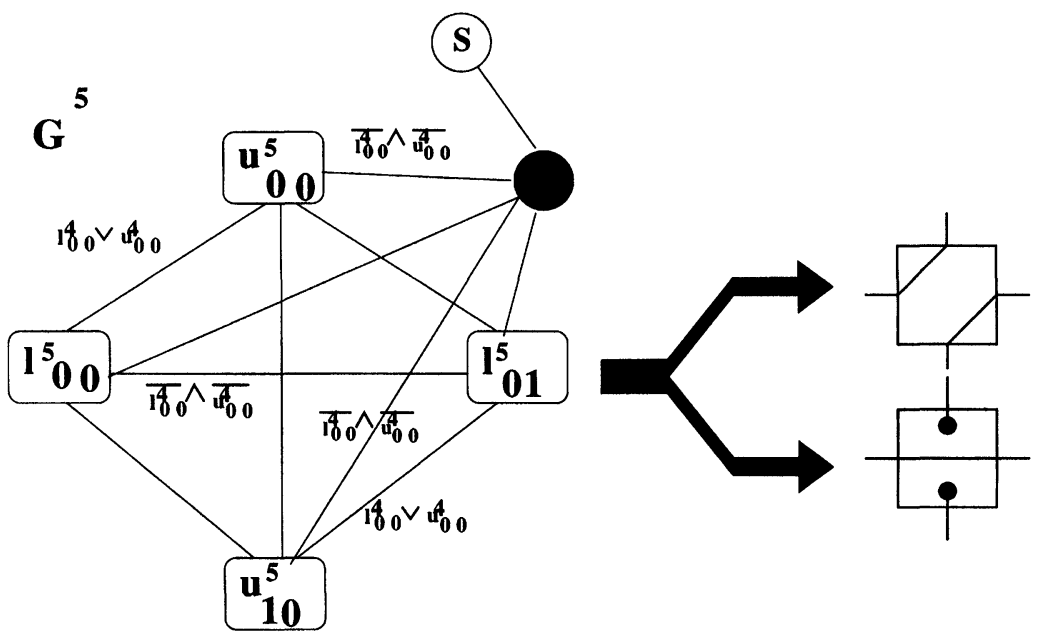

FIGURE 3 Edge-functions of processor $\mathrm{PE}_{0,0}$ resulting in two possible states. 


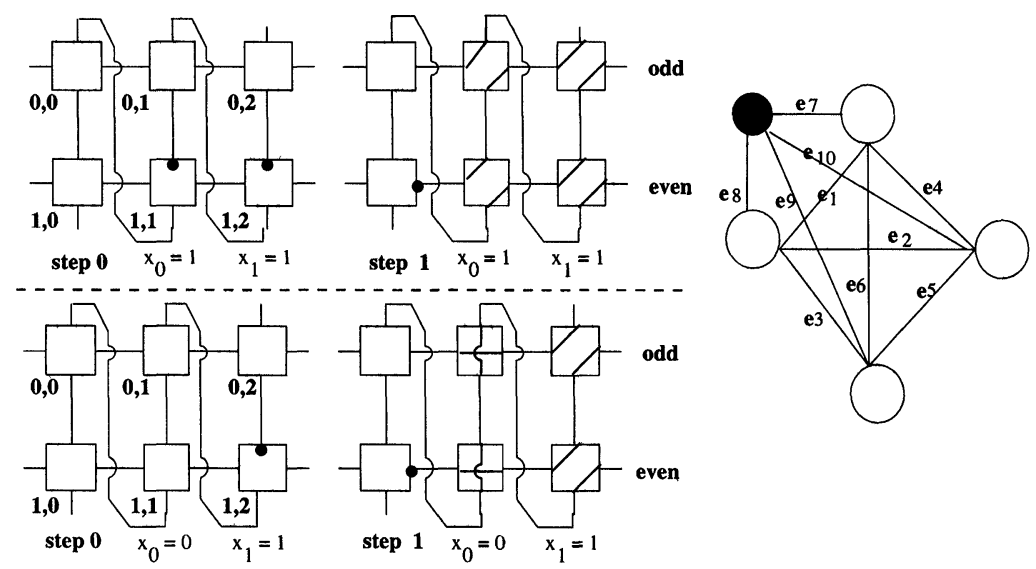

FIGURE 4 Two computations of the parity of 2-bit inputs. The edge-functions of processor $\mathrm{PE}_{0,0}$ induce two different states.

to the upper row. Next, each processor whose input bit is ' 1 ' chooses to redirect an incoming signal (broadcasted by $\mathrm{PE}_{0,0}$ ) to the upper row modulo two. If the input bit is ' 0 ' the processor chooses a state such that the incoming signal will remain in the same row. This can be easily decoded in our formal model by setting the value of the edge-functions as follows:

$$
\begin{gathered}
f_{\mathrm{e} 7_{1,1}}^{1}=x_{0} \quad f_{\mathrm{e} 7_{1,2}}^{1}=x_{1} \\
f_{\mathrm{e}_{0,1}}^{1}=f_{\mathrm{e}_{0,2}}^{1}=1 \quad f_{\mathrm{e} 10_{1,0}}^{2}=1 \\
\text { for } i=0,1 \quad j=1,2 \quad f_{\mathrm{e}_{i, j}}^{2}=f_{\mathrm{e}_{i, j}}^{2}=u_{i, j}^{1} \\
\text { for } i=0,1 \quad j=1,2 \quad f_{\mathrm{e}_{i, j}}^{2}=f_{\mathrm{e}_{i}, j}^{2}=\overline{u_{i, j}^{1}}
\end{gathered}
$$

while all other $f_{\mathrm{e}}^{t}$ are constant zero. It is easy to see that these edge functions correctly simulate the states assumed by the processors with respect to the input bits.

\section{THE CONSTRUCTION}

In this section we prove the main theorem, namely that a $T$ steps RN-MESH can always be simulated by a single step RN-MESH. The crux of the theorem is to construct a labeled graph that computes undirected connectivity of a special node $S$ and all other nodes in an input labeled graph.

The problem of undirected connectivity $\mathrm{UC}_{u, v}(G)$ is to decide if there is a path between $u$ and $v$ in $G . \overline{\mathrm{UC}}_{u, v}(G)$ is the complement function of $\mathrm{UC}_{u, v}(G)$, namely it outputs a 1 iff there is no path between $u$ and $v$ in $G$. In a labeled graph $G$, $G$. $\mathrm{UC}_{u, v}(G)=1$ when there is a path in $G$ between $u$ and $v$ and so that all the edge-functions along that path have the value of ' 1 '. Similarly, $\overline{\mathrm{UC}}_{u, v}(G)$ $=1$ when there is no such path in $G$ between $u$ and $v$.

Definition 3.1 Let $G^{1}, \ldots, G^{T}$ be a set of labeled graphs over $x_{1}, \ldots, x_{n}$ that represents a given RNMESH program $\mathrm{RN}_{N}^{T}$. We say that a labeled graph $G$ with (the same set of) inputs $x_{1}, \ldots, x_{n}$ simulates $G^{1}, \ldots, G^{T}$ iff there is a mapping of $G^{T}$, nodes to $G$ 's nodes $v \mapsto v^{\prime}$, such that for any value of $x_{1}, \ldots, x_{n}$, the value of $v^{\prime} \in G$ is the same as the value of $v \in G^{T}$.

TheOREM 3.1 Let $G^{1}, \ldots, G T$ be a set of labeled graphs over $x_{1}, \ldots, x_{n}$ that represents a given $R N-M E S H$ program $R N_{n}^{T}$. There exists a labeled graph $\hat{G}$ that simulates $G^{1}, \ldots, G^{T}$ with size $|\hat{G}| \leq$ $640^{T-1} \cdot n^{6 T-4}$.

Proof First, "complicated" edge-functions that query more than one variable are replaced by 
simple edge-functions of the form $f_{\mathrm{e}}^{t}=u_{i, j}^{t-1}$ or $f_{\mathrm{e}}^{t}=\overline{u_{i, j}^{t-1}}$, as follows:

IF $f_{\langle u, v\rangle}=r \wedge g$ then $\langle u, v\rangle$ is replaced by an auxiliary node $z$ and two consecutive edges $\langle u, z\rangle$ labeled by $r$ and $\langle z, v\rangle$ labeled by $g$.

IF $f_{\langle u, v\rangle}=r \vee g$ then $\langle u, v\rangle$ is replaced by two parallel edges: $\langle u, v\rangle$ labeled by $r$ and (another edge between the same vertices) $\langle u, v\rangle$ labeled by $g$.

Since in the case of the RN-MESH each edgefunction $f_{\mathrm{e} ? i, j}^{t}$ can query only four variables (those related to $\left.\mathrm{PE}_{i, j}\right)$, and there are 16 possible monomial in $f_{\mathrm{e}} ?_{i, j}$ 's DNF representation, each with 4 boolean variables, then the maximal number of variables of $f_{\mathrm{e} ?_{i, j}}$ is 64 (Some further optimization is omitted here, as it does not change the dominating factor). Hence, the total number of edges with edge-functions in the revised $G^{t}$ is not more than $10.64 \cdot n^{2}$, where $n^{2}$ is the size of the original RN-MESH. Note that this replacement does not change the value of nodes in $G^{t}$.

Next, we construct a new labeled graph $\hat{G}^{t-1}$ by modifying $G^{t}$ such that:

- The edges of $\hat{G}^{t-1}$ are labeled by edge-functions of $G^{t-1}$.

- There is a mapping from every node $v \in G^{t}$ to some node $\hat{v} \in \hat{G}^{t-}$, so that the value $v$ (when evaluating the sequence $G^{1}, \ldots, G^{t}$ ) is the same as that of $v^{\prime}$ (when evaluating the sequence $G^{1}, \ldots, G^{t-2}, \hat{G}^{t-1}$.

The construction of $\hat{G}^{t-1}$ depends on the type of its edge-functions $f_{\mathrm{e} ? i, j}^{t}$ :

$f_{\mathrm{e} ? i, j}^{t}=u_{i^{\prime}, j^{\prime}}^{t-1}$ so that in terms of connectivity $f_{\mathrm{e} ? i, j}^{t}=\mathrm{UC}_{S, u_{i^{\prime}, j^{\prime}}^{t}}\left(G^{t-1}\right)$, (and similarly for $\left.l_{i^{\prime}, j^{\prime}}^{t}\right)$. In this case, the edge $\mathrm{e}{ }_{i, j}=\langle\alpha, \beta\rangle$ is replaced by a copy of $G^{t-1}$ such that $\alpha$ is identified with $S$ of $G^{t-1}$ and $\beta$ is identified with $u / l_{i^{\prime}, j^{\prime}}^{t}$. It follows that the value of the nodes $\alpha$ and $\beta$ in $\hat{G}^{t-1}$ is the same as in $G^{t}$.

$f_{\mathrm{e} ?_{i, j}}^{t}=\overline{u_{i^{\prime}, j^{\prime}}^{t-1}}$ where in terms of connectivity $f_{\mathrm{e} ?, j}^{t}=\overline{\mathrm{UC}}_{S, u_{i^{\prime}, j^{\prime}}^{t-1}}\left(G^{t-1}\right.$ ) (and similarly for $l_{i^{\prime}, j^{\prime}}^{t}$ ). In this case, the edge $\mathrm{e} ?_{i, j}=\langle\alpha, \beta\rangle$ is replaced by $\overline{G_{S, u / l}^{t-1} i^{\prime}, j^{\prime}}$, which is the labeled graph that computes
$\overline{\mathrm{UC}}_{S, u / l_{l^{\prime}, j^{\prime}}^{t-1}}\left(G^{t-1}\right)$. As Lemma 4.1 will show, $\overline{G_{S, u / l^{\prime}, j^{\prime}}^{t-1}}$ can be constructed with respect to $u / l_{i^{\prime}, j^{\prime}}^{t-1}$ with a pre-designated node $v^{\prime}$ such that $v^{\prime}=1$ in $\overline{G_{S, u / l}^{t-1} t-1}$ iff $u / l_{i^{\prime}, j^{\prime}}^{t-1}=0$ in $G^{t-1}$. In this case $\alpha$ is identified with $S$ of $\overline{G_{S, u / l^{\prime}, j^{\prime}}^{t-1}}$ and $\beta$ is identified with $v^{\prime}$. By the definition of $\frac{G^{t, j},-1}{G_{S, u / l^{t-1}, l^{\prime}}}$ the value of the nodes $\alpha$ and $\beta$ in $\hat{G}^{t-1}$ is the t $^{i^{\prime}, \text { same }^{\prime}}$ as in $G^{t}$. Lemma 4.1 guarantees that the size (number of edges) of $\overline{G_{S, u / l^{\prime}, j^{\prime}}^{t-1}}$ satisfies that $\left|\overline{G_{S, u / l_{l^{\prime}, j^{\prime}}^{t-1}}^{t-1}}\right| \leq n^{4}\left|G^{t-1}\right|$ (see Section 4 for a detailed construction).

The total size expansion needed to create $\hat{G}^{t-1}$ can be bounded by assuming the worst case in which all $640 \cdot n^{2}$ internal edges of $G^{t}$ have been labeled by edge-function $f_{\mathrm{e} ?_{i, j}}^{t}=\overline{u / l_{i^{\prime}, j^{\prime}}^{t}}$. Thus, in the worst case $\left|\hat{G}^{t-1}\right| \leq 640 \cdot n^{2}\left(n^{4} \cdot\left|G^{t-1}\right|\right)$.

The construction described above is repeated $T-1$ times:

$$
G^{T} \rightarrow \hat{G}^{T-1} \rightarrow \hat{G}^{T-2} \cdots \rightarrow \hat{G}^{1}=\hat{G} .
$$

The resulting graph $\hat{G}$ can be evaluated directly from $x_{1}, \ldots, x_{n}$, (i.e., the value of the boolean variables associated with its nodes are functions of $\left.x_{1}, \ldots, x_{n}\right)$, yet it performs the same computation as the original sequence $G^{1}, \ldots, G^{T}$. The size of $\hat{G}$ is obtained by bounding the size blowup in each of the $T-1$ applications of the construction as described above, yielding

$$
|\hat{G}| \leq 640^{T-1} \cdot n^{6 T-4}
$$

We proceed to show that the labeled graph $\hat{G}$ can be simulated by a single step RN-MESH.

COROllary 3.2 Given $R N_{n}^{T}$ with inputs $x_{1}, \ldots, x_{n}$ there exists a reconfigurable mesh $R N_{640^{2(T-1)} \cdot n^{12 T-8}}^{1}$ that simulates $R N_{n}^{T}$ in one step.

Proof Let $\mathrm{G}^{1}, \ldots, G^{T}$ represent $\mathrm{RN}_{n}^{T}$, and let $\hat{G}$ be the labeled graph that simulates $G, \ldots, G^{T}$ using the construction of Theorem 3.1. Then the RNMESH that simulates $\hat{G}\left(\mathrm{RN}_{640^{2(T-1)} \cdot n^{12 T-8}}^{1}\right)$ can be 
constructed by implementing the adjacency matrix of $\hat{G}$. This is a standard technique (see for instance $[2,6])$ and is commonly used to find connected components of an undirected graph in a single step.

Basically, we take an RN-MESH of the size of the adjacency matrix, and initially connect every column and every row and the diagonal (so that the $i$-th column is connected to the $i$-th row). In addition, the $(i, j)$-th processor connects the $i$-th row to the $j$-th processor if the $i$-th and the $j$-th nodes in $\hat{G}$ are connected by an edge of which the edge-function is evaluated to ' 1 '. By the construction of Theorem 3.1 the edge functions are of the form $x_{i}$ or $\bar{x}_{i}$, hence the configuration can be set instantly. Once the configuration is set, the leftmost processor in the row of the special node $S$ transmits a ' 1 ' which is received by all processors in its connected component, and in particular by those in the rows that are mapped to output processors of $R N_{n}^{T}$ of the original computation. The left most processor in each of these rows now outputs the result.

Using the adjacency matrix technique as described above require an RN-MESH of size that is quadratic in the size of $\hat{G}$.

\section{COMPUTING $\overline{\mathrm{UC}}_{s, t}(G)$}

Following the technique of Nisan and Ta-Shma [13] we see in this section that $\overline{\mathrm{UC}}_{s, t}(G)$ of an undirected graph $G$ can be computed by an almost monotone boolean formula (using only $\wedge, \vee$ ) whose varaibles are connectivity queries on nodes in $G$. The method is a monotone reduction from $\overline{\mathrm{UC}}_{s, t}(G)$ to $\mathrm{UC}_{s, t}(G)$. For the sake of completeness and to show exact leading constants, we give here a brief review of the technique. However, some significant changes were made in order to fit this method to our needs. In particular, the AKS sorting network used in [13] to pack non-zero elements is replaced by an explicit formula for computing the $i$-th bit in the packed vector of $\left[x_{1}, \ldots, x_{n}\right]$ :

$$
\begin{aligned}
\operatorname{pack}_{i}\left(\left[x_{k}, \ldots, x_{n}\right]\right) & \\
= & \begin{cases}x_{k} & i=1 \\
0 & i>n-k \\
\left(\operatorname{pack}_{i-1}\left(\left[x_{k+1}, \ldots, x_{n}\right]\right) \wedge x_{k}\right) & \\
\quad \vee \operatorname{pack}\left(\left[x_{k+1}, \ldots, x_{n}\right]\right) & i \leq n-k\end{cases}
\end{aligned}
$$

Clearly the size of pack $_{i}$ is less than $2 n^{2}$.

Hereafter, we assume that the nodes of $G$ are numbered from 1 to $n$, and denote $\vee_{i=1}^{n} f_{i}=$ $f_{1} \vee \cdots \vee f_{n}$ and $\wedge_{i=1}^{n} f_{i}=f_{1} \wedge \cdots \wedge f_{n}$. The complete formula of $\overline{\mathrm{UC}}_{s, t}(G)$ is derived as follows:

$\overline{U C}_{s, t}(G)=\vee_{i=2}^{n} \mathrm{NCC}_{i}(G) \wedge \mathrm{NCC}_{i-1}\left(G^{+}\right) \quad$ where $G^{+}$is the graph obtained by adding an edge between $s$ and $t$ and $\mathrm{NCC}_{i}(G)=1$ iff the number of connected components in $G$ is $i$, otherwise $\mathrm{NCC}_{i}(G)=0$. If the new edge added to $G$ caused the number of connected components in $G^{+}$to decrease, then clearly $s$ and $t$ were disconnected in G. Since we forbid negations, we must scan all possible values of $i$.

$\operatorname{NCC}_{i}(G)=f_{i+1}(G) \wedge g_{n-i+1}(G)$ such that if $k$ is the number of connected components in $G$, then $f_{i}(G)=1$ iff $k<i$ and $g_{i}(G)=1$ iff $n-k<i$ or $k>n-i$. Thus, the only value of $i$ for which both functions agree is when $i=k \neq \#$ components in $-G$.

$f_{i}(G)=\operatorname{pack}_{i}\left[L I_{1}(G), \ldots, L I_{n}(G)\right]$ where $L I_{j}(G)=$ 0 iff $j$ is the largest node in its connected component. Thus $\left\langle f_{1}(G), \ldots, f_{n}(G)\right\rangle$ contains a ' 0 ' for every connected component in $G$.

$\operatorname{LI}_{i}(G)=\vee_{j=i+1}^{n} U C_{i, j}(G)$ so that $\operatorname{LI}(G)=1$ iff there is a node with a greater index than $i$ which is connected to $i$.

$g_{j}(G)=\operatorname{pack}_{i}\left(\left[S F_{1,1}(G), \ldots, S F_{n, n}(G)\right]\right) \quad$ where $S F_{k, j}(G)=0$ iff the edge $\langle k, j\rangle$ belongs to the first lexicographic spanning forest of $G$. Thus $g_{i}(G)=1$ iff there are more than $i$ edges in the first lexicographic spanning forest of $G$ and less than $n-i$ connected components in $G$ [13]. (The key idea here is that the size of the spanning forest is the complement to the number of connected components, making monotone counting of the number of connected components possible). 
$\mathrm{SF}_{i, j}(G)=\mathrm{UC}_{i, j}(G) \vee\langle i, j\rangle \notin G$ so that $\mathrm{SF}_{i, j}(G)$ $=1$ iff $\langle i, j\rangle$ does not belong to the first lexicographic forest of $G$. Note that $\langle i, j\rangle \notin G$ is a nonmonotone query; however, it can be viewed as connectivity query over the graph with two vertices $(i$ and $j$ ). This point is further explained in the sequel.

Counting the total number of $\mathrm{UC}_{s, t}(G)$ queries of the resulting formula yields that the total size of the formula is less than $5 n^{4}$ connectivity queries $\left(\mathrm{UC}_{i, j}(G)\right)$.

The above formula can be directly used to construct the complement labeled graph $\bar{G}$ :

LemMA 4.1 Let $\mathrm{G}$ be a labeled graph with $n$ nodes over the boolean variables $x_{1}, \ldots, x_{n}$ and a predesignated node $v \in G$. Then three exists a labeled graph $\bar{G}$ over $x_{1}, \ldots, x_{n}$ with a pre-designated node $v^{\prime}$ such that the value of $v^{\prime}$ in $\vec{G}$ is one iff the value of $v$ in $G$ is zero. The size of $\bar{G}$ is polynomial in the size of $G$ and satisfies that $|\bar{G}| \leq n^{4}|G|$.

Proof Note that $\bar{G}$ actually computes $\overline{\mathrm{UC}}_{S, v}\left(G^{\prime}\right)$, where $G^{\prime}$ is the graph obtained after removing the edges in $G$ whose edge-functions evaluated to zero. Thus we actually need the formula to compute undirected connectivity in $G^{\prime}$. Now, let $G_{f}$ denote a labeled graph that computes $f\left(x_{1}, \ldots, x_{n}\right)$ with a special output node $v_{f} \in G_{f}$, such that the boolean variable associated with $v_{f}$ is 1 iff $f\left(x_{1}, \ldots, x_{n}\right)=1$. The formula of $\overline{\mathrm{UC}}_{S, v}$ can be recursively constructed as follows. Note that the inputs to this formula are connectivity queries of the form $G_{\mathrm{UC}_{s, t}(G)}$ and "edgemembership" queries of the form $G_{\langle i, j\rangle \notin G}$.

$G_{f \wedge g}$ can be constructed by identifying $S$ of $G_{g}$ with $v_{f}$, letting $v_{g}$ be the special output node of $G_{f \wedge g}$. The source node of $G_{f \wedge g}$ should be the source node of $G_{f}$. Clearly $G_{f \wedge g}$ computes $f\left(x_{1}, \ldots, x_{n}\right) \wedge g\left(x_{1}, \ldots, x_{n}\right)$.

$G_{f} \vee g$ can be constructed by identifying the source nodes of $G_{f}$ and $G_{g}$ forming the source node of $G_{f \vee g}$. A new node $v_{f \vee g}$ that is connected to both $v_{f}$ and $v_{g}$ is added, forming the output node of $G_{f} \vee g$. Clearly $G_{f \vee g}$ computes $f\left(x_{1}, \ldots, x_{n}\right) \vee g\left(x_{1}, \ldots, x_{n}\right)$.
$G_{\mathrm{UC}_{s, t}(G)}$ is formed from $G$ using $s$ as the source node and $t$ as the output node of $G_{\mathrm{UC}_{s, t}(G)}$.

$G_{\langle i, j\rangle \notin G}$ can be constructed according to the edge $\langle i, j\rangle$ as follows:

- If $\langle i, j\rangle \in G$ and $\langle i, j\rangle$ has no edge-function, then $G_{<i, j>\notin G}$ contains two nodes $i$ and $j$ as source and output with no edge between them

- If $\langle i, j\rangle \in G$ and $\langle i, j\rangle$ has an edge-function $f$, then $G_{<i, j>\notin G}$ contains two nodes $i$ and $j$ as source and output nodes, and an edge that connects them with the edge-function $\bar{f}$.

- If $\langle i, j\rangle \notin G$ then $G_{\langle i, j\rangle \notin G}$ contains two nodes and $i$ and $j$ as source and output and an edge between them.

The validity of this construction and its size are due to its being a direct simulation of the monotone circuit of [13].

\section{CONCLUSIONS}

In this work we have shown a general construction so that a $T$ steps RN-MESH of size $n \times n$ can be simulated by a single step RN-MESH, where the corresponding mesh size increase by a factor of $n^{O(T)}$. The construction itself is simpler and smaller as compared to a similar result previously obtained for the directed model [5].

A previous work that gave self-simulation of large meshes on smaller ones may be seen as the complement of the current paper [2]. Combining these two results yield a complete scalability framework in terms of a Time-Size Tradeoff for computing with reconfigurable meshes. Namely, if more hardware is available so that the mesh is large then computation can be accelerated. In the opposite direction, one may always scale his algorithm to a smaller mesh at the expense of slowing down the algorithm.

Interestingly, one consequence of this result is that whatever the definition of a complexity class for the reconfigurable mesh involves polynomial size meshes (as, for example, is the case in [3]), it is 
insensitive to the precise number of steps required to solve a problem. For specific problems it makes sense to give tradeoffs of the form timed time $^{c_{1}} \cdot \operatorname{size}^{c_{2}} \geq f(n)$ (where $n$ is the size of the input). In fact, it was previously observed that the RN-MESH follows the Thompson VLSI model, and thus $A T^{2}$-type lowerbounds in the Thompson model apply to it [16]. More work is required in this direction, e.g., when the power of broadcasts is restricted to a certain "length" of the bus.

Finally, this work leaves a lot of space for further research. One may derive bounds on the minimal number (as a constant) of steps that specific problems require when the size of the mesh remains fixed. Further effort may find ways to improve our general construction in terms of the blowup in the size of the mesh. One may want to give explicit single step constructions for known reconfigurable algorithms which use reduced size than that required by the general construction.

\section{References}

[1] Alnuweiri, H., Alimuddin, M. and Aljunaidi, H., Switch models and reconfigurable networks: Tutorial and partial survey. In: Reconfigurable Architecture Workshop. IEEE, April, 1994.

[2] Ben-Asher, Y., Gordon, D. and Schuster, A. (1995). Efficient self simulation algorithms for reconfigurable arrays. Journal of Parallel and Distributed Computing, 30, $1-22$.

[3] Ben-Asher, Y., Lange, K.-J., Peleg, D. and Schuster, A. (1995). The Complexity of Reconfiguring Network Models. Information and Computation, 121(1), 41-58.

[4] Ben-Asher, Y., Peleg, D., Ramaswami, R. and Schuster, A. (1991). The Power of Reconfiguration. Journal of Parallel and Distributed Computing, 13(2), 139-153. Special Issue on Massively Parallel Computation.

[5] Ben-Asher, Y. and Schuster, A. (1996). Time-Size Tradeoffs for Reconfigurable Meshes. Parallel Processing Letters, 6(2), $231-245$.

[6] Hayashi, T., Nakano, K. and Olariu, S., Efficient List Ranking on the Reconfigurable Mesh, with Applications. Technical Report TR96-03, Hayashi Lab, May 1996. To appear Proc. ISAAC' 96.

[7] Immerman, N. (1988). Nondeterministic space is closed under complementation. SIAM J. on Computing, 17(5), 935-938.

[8] Jang, J., Park, H. and Prasanna, V. K., A bit model of reconfigurable mesh. In: Reconfigurable Architecture Workshop. IEEE, April, 1994.

[9] Jang, J. and Prasanna, V. K. (1992). An optimal sorting algorithm on reconfigurable mesh. In: Proc. Inter. Parallel Processing Symp., pp. 130-137.
[10] Miller, R., Prasanna, V. K., Reisis, D. I. and Stout, Q. F. (1991). Image Processing on reconfigurable meshes. In: From Pixels to Features II, North-Holland, pp. 85-101.

[11] Miller, R., Prasanna-Kumar, V. K., Reisis, D. I. and Stout, Q. F. (1987). Parallel computations on reconfigurable meshes. Technical Report TR IRIS\#229, Dept. of Computer Science, University of Southern California. To appear in IEEE Transactions on Computers.

[12] Nigam, M. and Sahni, S., Sorting $n$ numbers on $n \times n$ reconfigurable meshes with buses. In: Proc. 7th International Parallet Processing Symposium,pp. 174-181. IEEE, April, 1993.

[13] Nisan, N. and Ta-Shma, A. (1992). Symmetric Logspace is Closed Under Complement. In: 24th Ann. ACM STOC, pp. 619-623.

[14] Olariu, S. and Schwing, J.-L. (1996). A New Deterministic Sampling Scheme with Applications to Broadcast-Efficient Sorting on the Reconfigurable Mesh. Journal of Parallel and Distributed Computing, 32, 215-222.

[15] Jerry, L., Trahan, R., Thiruchelvan, K. and Vaidyanathan, R., On the power of segmenting and fusing buses. In: Proceedings of 7th International Parallel Processing Symposium, pp. 79-83, IEEE, April, 1993.

[16] Schuster, A., Dynamic Reconfiguring Networks for Parallel Computers: Algorithms and Complexity Bounds. Ph.D. Thesis, Hebrew University, Jerusalem, ISRAEL, August 1991.

[17] James, L. (1993). Schwing Stephan Olariu and Jingyuan Zhang. Applications of reconfigurable meshes to constant time computations. Parallel Computing, 19, 229-237.

[18] Wang, B. and Chen, G. (1990). Constant time algorithms for the transitive closure and some related graph problems on processor arrays with reconfigurable bus sytems. IEEE Transactions on Parallel and Distributed Systems, 1(4), 500-507.

\section{Author's Biographies}

Assaf Schuster Assaf Schuster received his Ph.D. in Computer Science from the Hebrew University of Jerusalem in 1991. He is currently a senior lecturer at the Technion-Israel Institute of Technology. His interests include distributed shared memory, high-performance computing on non-dedicated clusters of PCs, networks and routing algorithms, optical computation and communication, and dynamically reconfiguring networks. His main current project is MILLI-PEDE: an all user mode, no kernel-patches, "add on" software tool for standard corporate environments, that takes advantage of idle system resources and efficiently utilizes idle processor time in available distributed environments of personal workstations. His home page can be found in http://www.cs.technion.ac.il/ $\sim$ assaf. 
Yosi Ben-Asher Yosi Ben-Asher received his Ph.D. in computer science from the Hebrew University in 1989 . He is currently a lecturer at the Department of Mathematics and Computer Science, University of Haifa, Israel. His research interests include parallel systems, parallel languages, compilers, and reconfigurable networks. His main current project is PCOMP: a compiler for Distributed Shared Memory systems. 

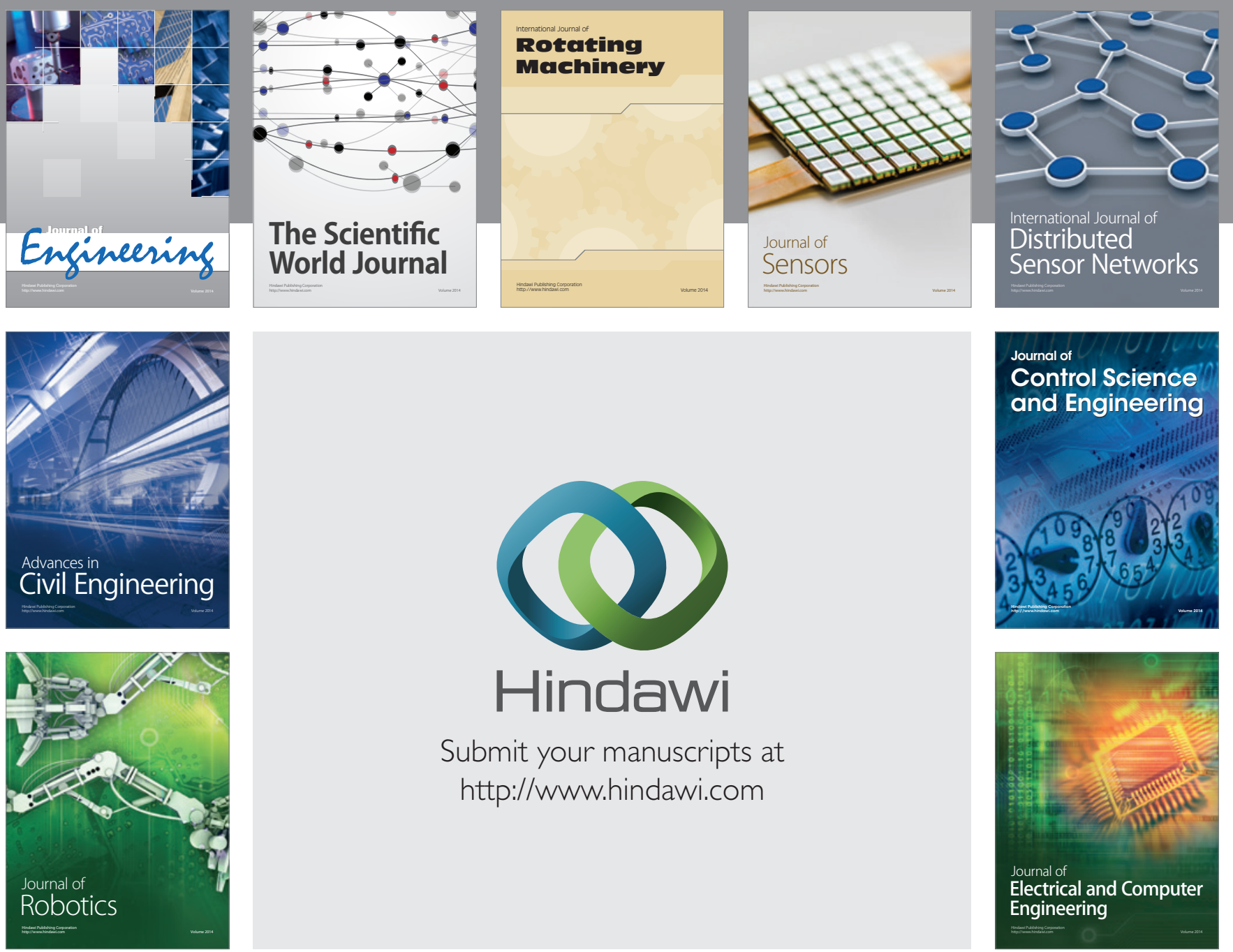

Submit your manuscripts at

http://www.hindawi.com
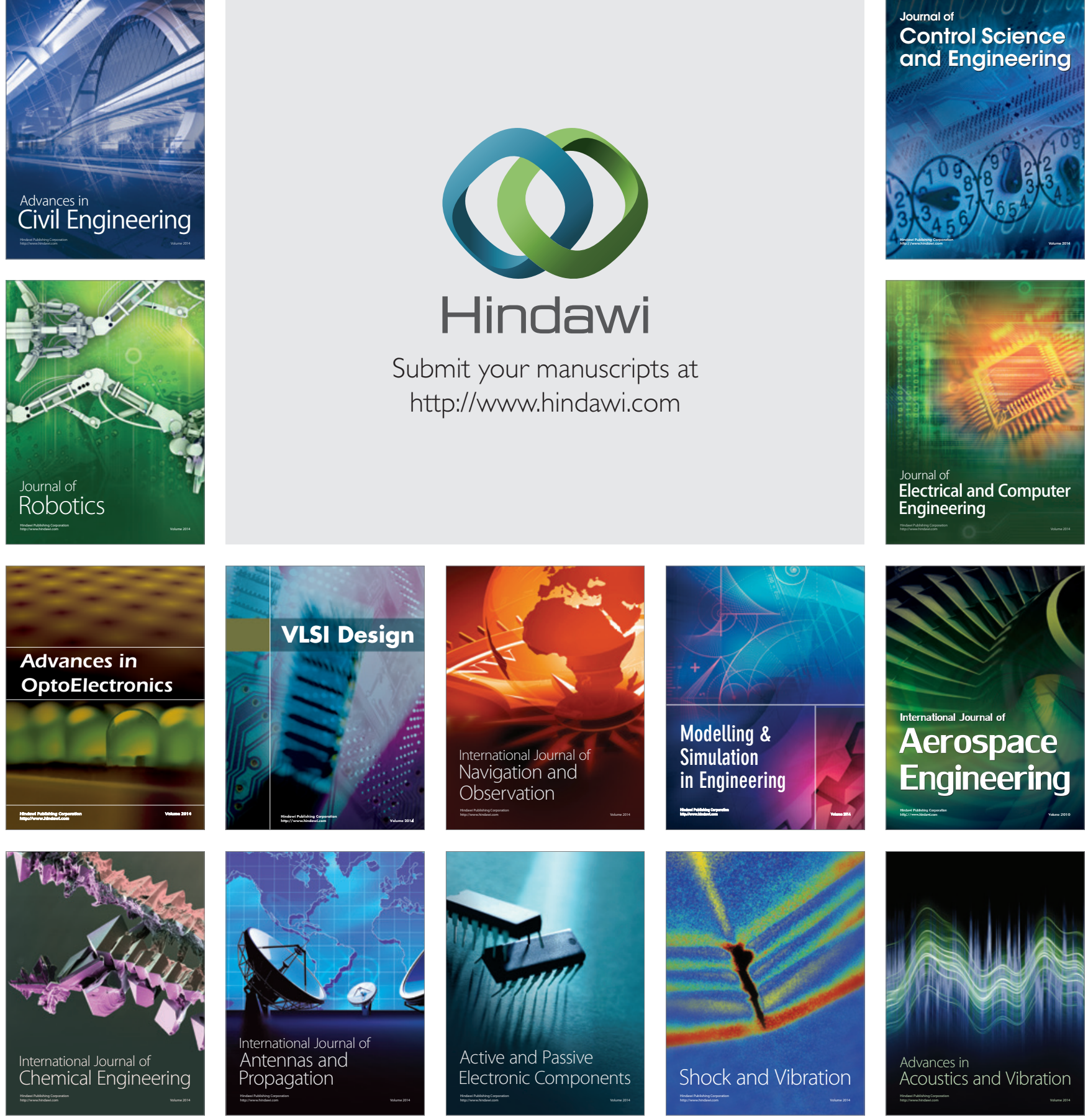